\title{
Factors of Health, Quality of Life and Well-Being of Young Indigenous Peoples of the North, Siberia and the Far East of the Russian Federation
}

\author{
Sergey Trapitsin*, Elena Agapova, Olga Granichina, Marina Zharova
}

\author{
Herzen State Pedagogical University of Russia, St. Petersburg, Russia \\ *Corresponding author. Email: trapitsin@gmail.com
}

\begin{abstract}
The data on the health of the indigenous minorities of the North, Siberia and the Far East of the Russian Federation, assesses its impact on the quality of life and subjectively perceived well-being, draws attention to the lack of objective information obtained exclusively from statistical reports are analyzes in the paper. The correlation of health indicators with the effectiveness of measures to improve the quality of medical services is noted. In conclusion, it is concluded that the concept of health has changed, as well as the need for regular monitoring of the quality of life of the indigenous minorities, not only as a factor of sustainable development of territories, but also as a condition for preserving their population.
\end{abstract}

Keywords: indigenous peoples, health factors, quality of life.

\section{INTRODUCTION}

Improving the quality of life of the indigenous minorities of the North, Siberia and the Far East, primarily children and youth, is among the problems of the development of the northern territories of the Russian Federation, which today arouse increased interest among politicians, economists, sociologists, researchers and public [4].

The quality of life means the degree of development and the completeness of satisfaction of the entire complex of their needs and interests, the level of comfort of the social and natural environment for human life in a particular territory, the degree of preservation of the language, culture and traditions that determine the level well-being, social, spiritual and physical health of a person [1].

Indicators of the quality of life are determined by approaches to its assessment, which imply, inter alia, an assessment of the effectiveness of specially planned and implemented measures to improve it: the construction of infrastructure facilities, ensuring the availability of education, high-quality medical services, support for traditional types of economic activity, etc. [8].

For the indigenous minorities of the North, Siberia and the Far East of the Russian Federation the "quality of life" concept, in addition to generally accepted and wellknown indicators, includes the possibility of creating communities for engaging in traditional trades, crafts, preserving and reviving national traditions, language, writing, etc.

The nature and content of state policy in this region is largely determined by its uniqueness, primarily by the fact that, occupying almost $70 \%$ of the area of Russia, and accounting for only about $7 \%$ of its population, more than 100 nationalities live on its territory, 40 of which make up the indigenous minorities of the North, Siberia and the Far East, included in the list approved by the Government. Behavioral models, conditioned by the climate and peculiarities of the nature of the North, have been formed among these peoples for hundreds of years and have survived almost in their original form. Today, the indigenous minorities of the North, Siberia and the Far East are under the patronage of the Federal Protection Program, which is comprehensive and focused on various aspects of improving the quality of their lives [3].

\section{MATERIALS AND METHODS}

Russia has accumulated significant empirical data on the socio-economic health factors of the indigenous minorities of the North (Markin, Silin, Voronov, 2019; Kozlov, Vershubsky, Kozlova, 2007). At the same time, 


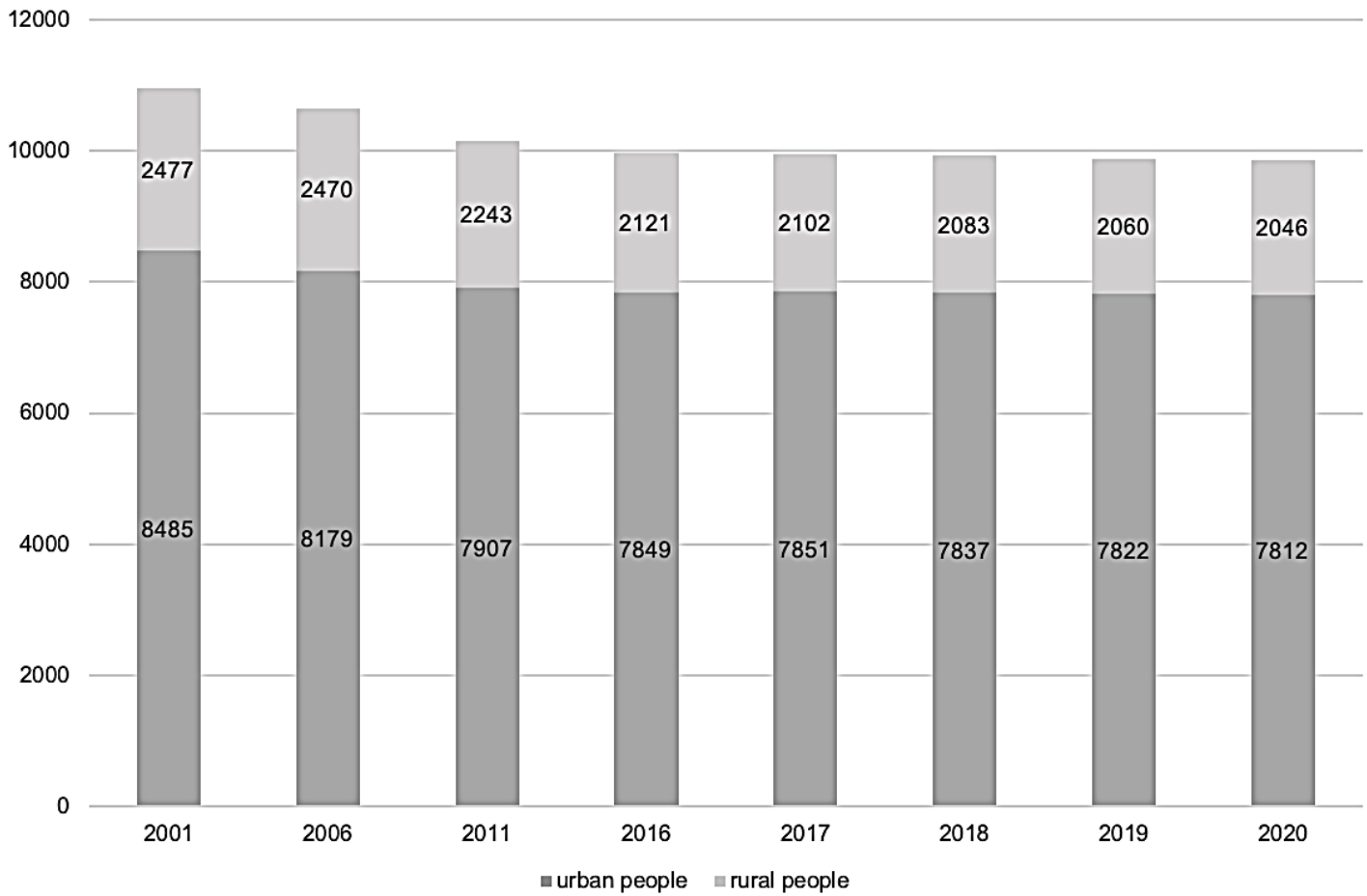

Figure 1 Resident population dynamics, 2001-2020

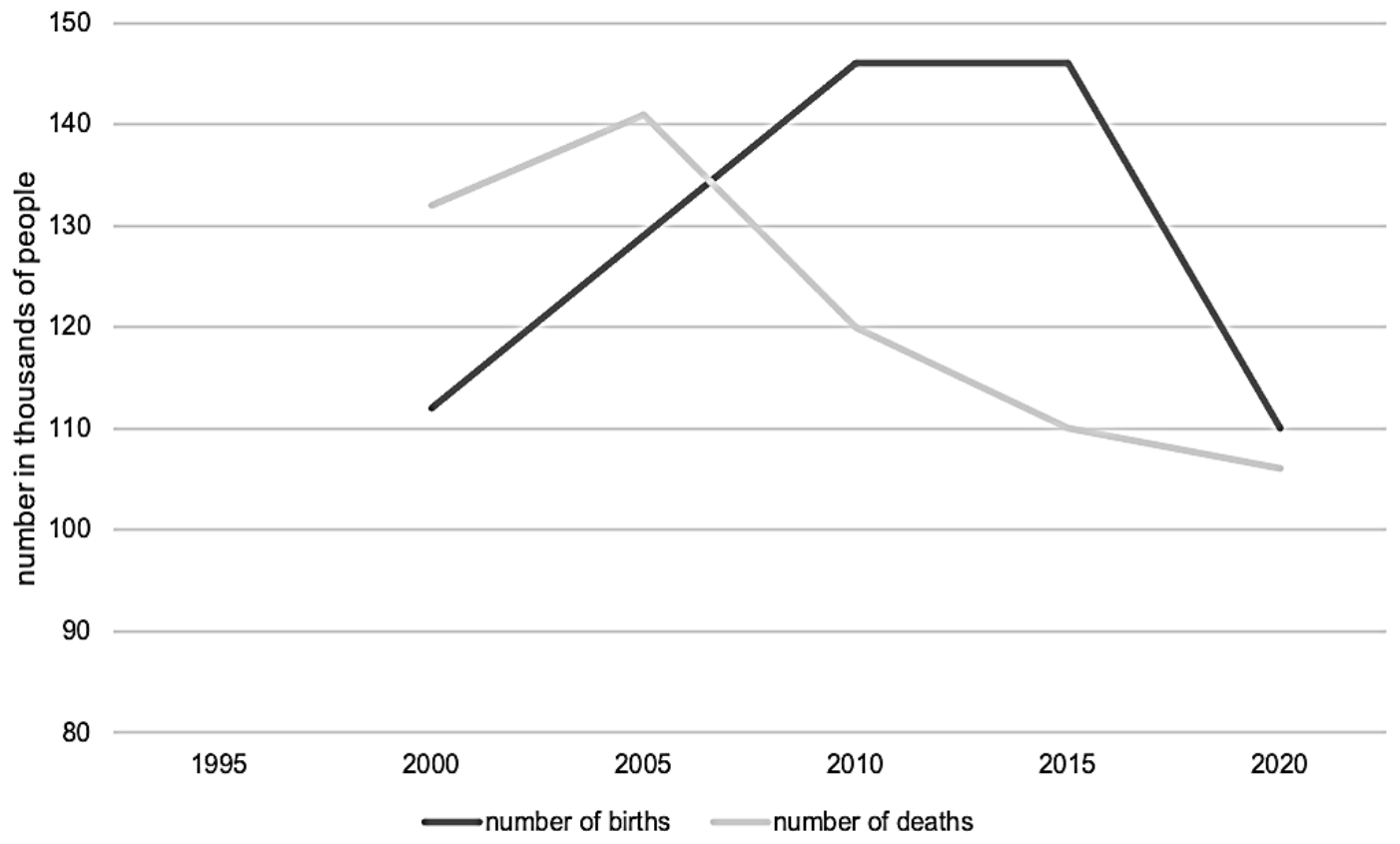

Figure 2 Dynamics of birth and death rates

this information is mostly the result of scientific research, and not the data of official statistics, which forms the basis for developing policies and making management decisions [6].

The use of comparable indicators in different constituent entities of the Russian Federation makes it possible to correctly compare the key indicators of the health and quality of life of the indigenous minorities of the North, Siberia and the Far East with representatives of other ethnic groups living in the same territories of the Russian Federation, highlighting the territorial and ethnic specifics.

Quantitative data and indicators characterizing the state and development trends of the health care system in 


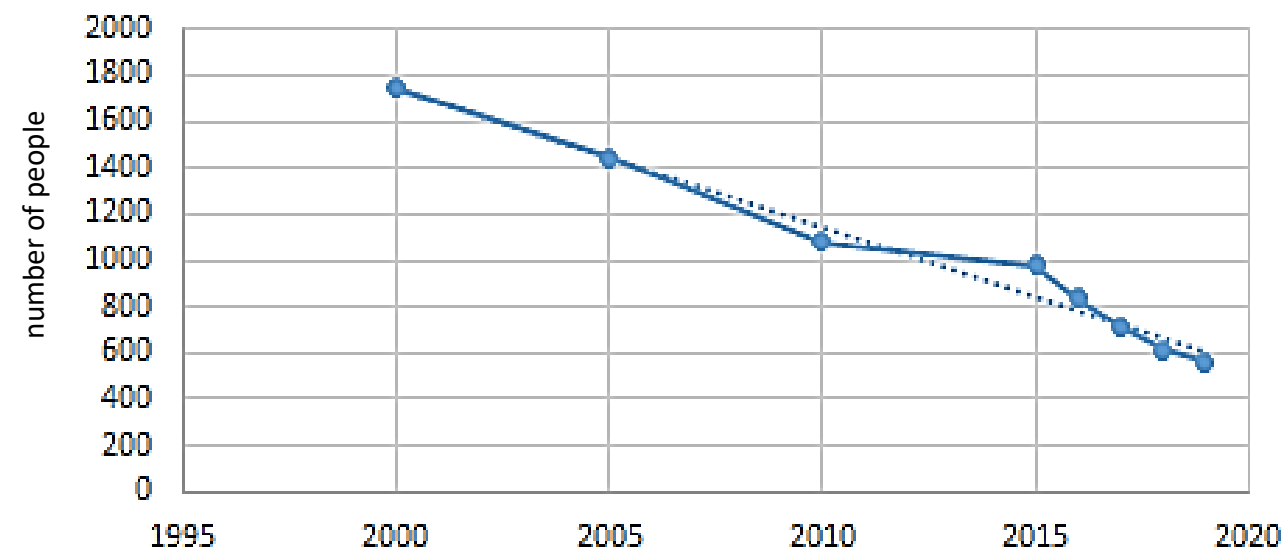

Figure 3 Infant mortality rates.

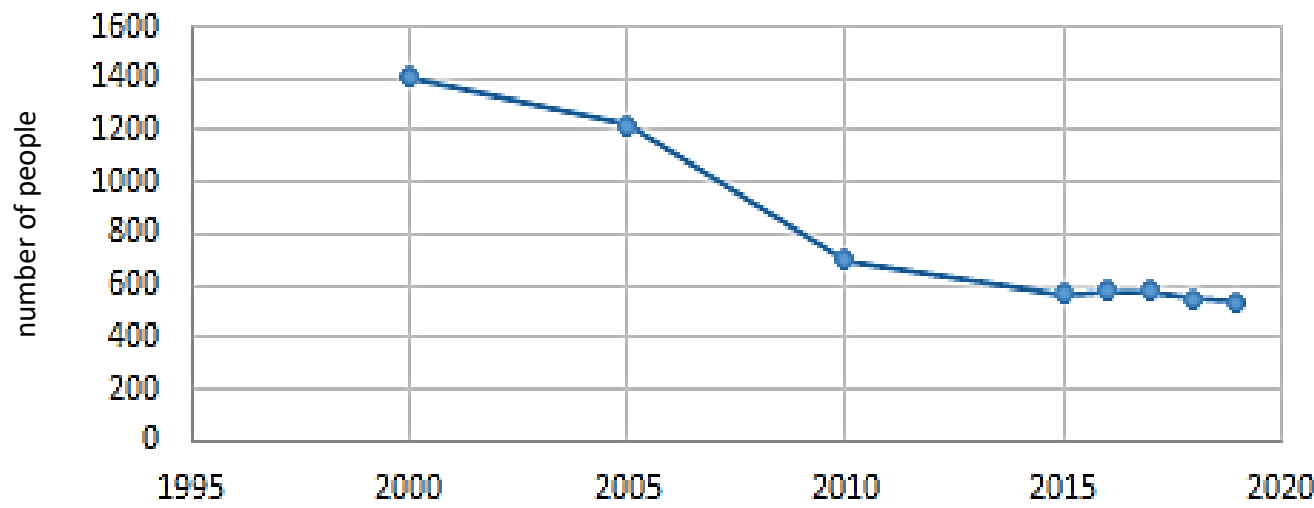

Figure 4 The number of alcoholism diseases, 2000-2019.

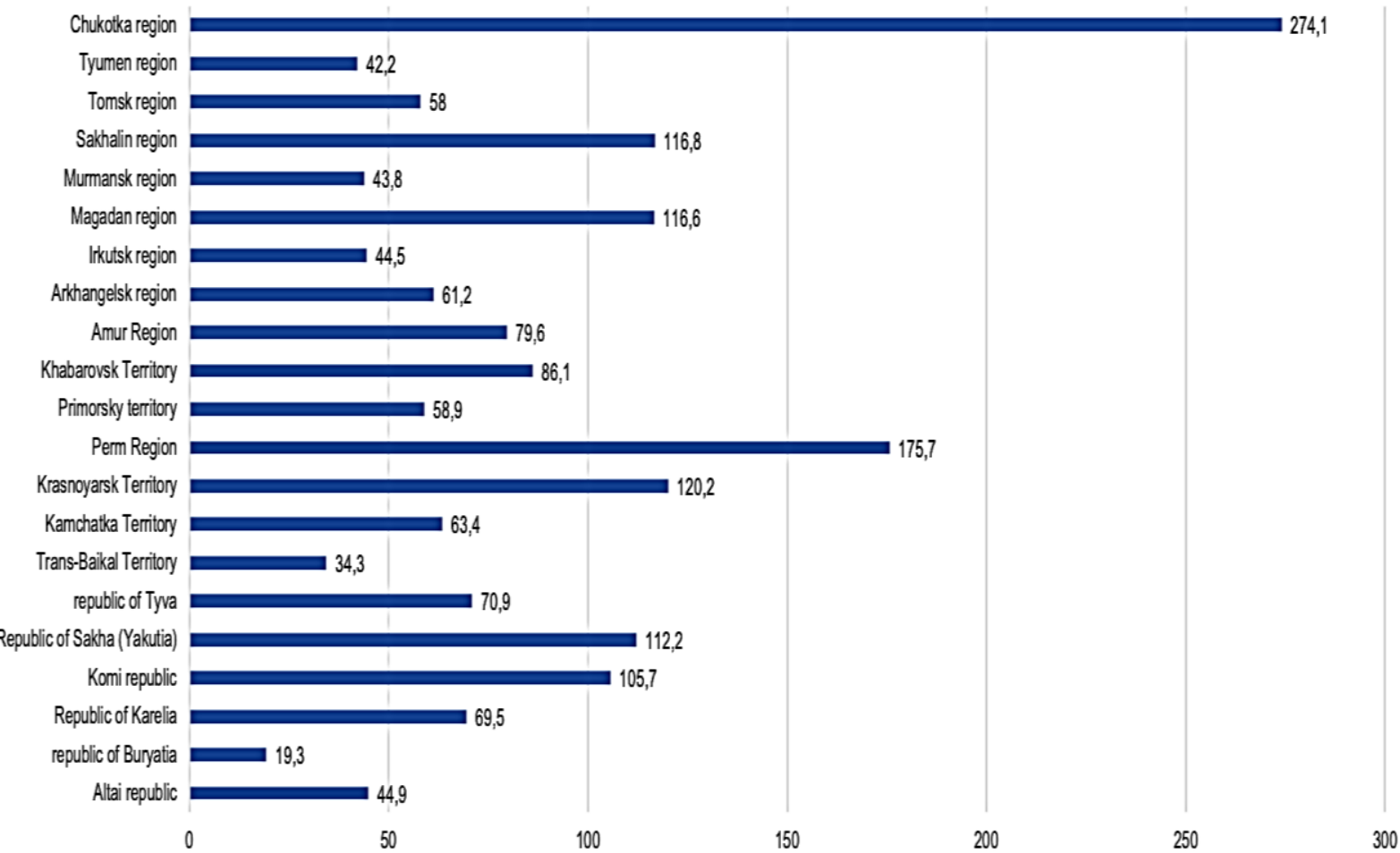

Figure 5 The number of alcoholism and alcoholic psychosis diseases by region, 2019 


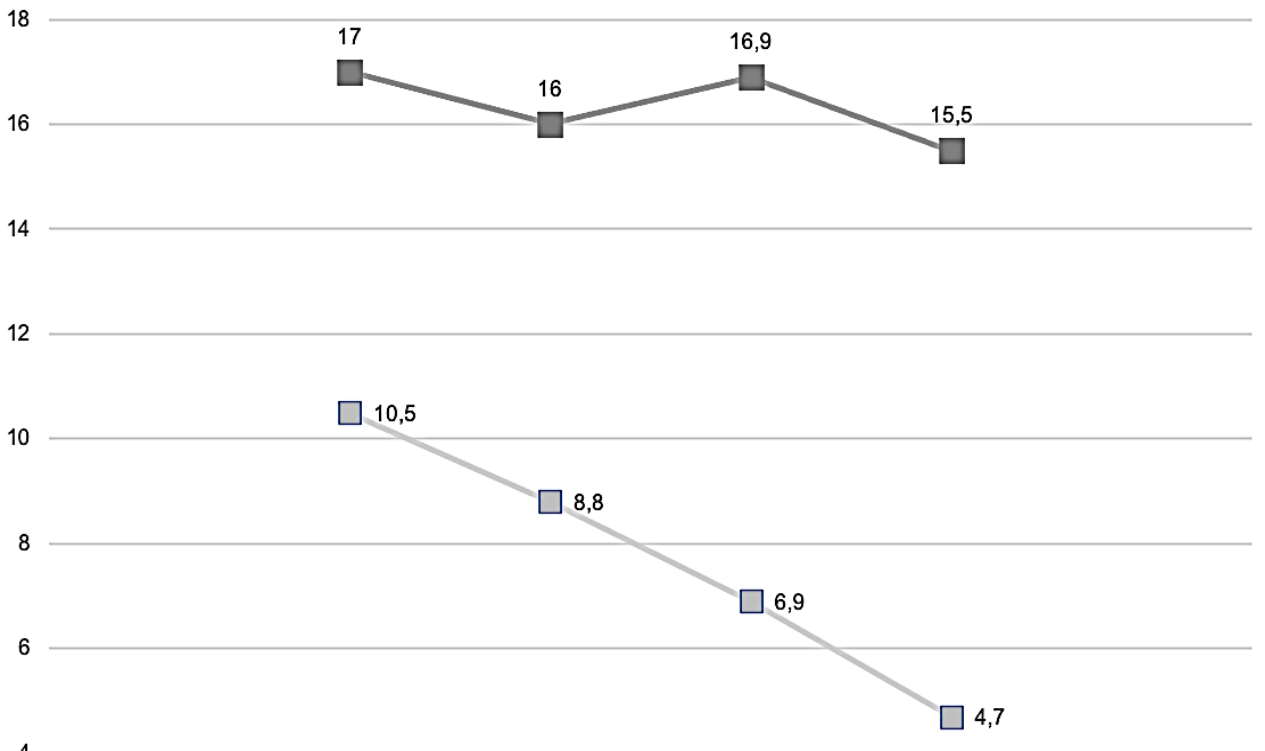

2

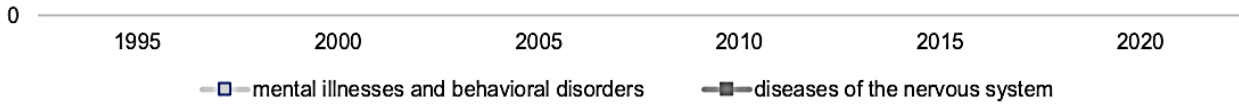

Figure 6 Dynamics of the number of mental illnesses and diseases of the nervous system, 2000-2019

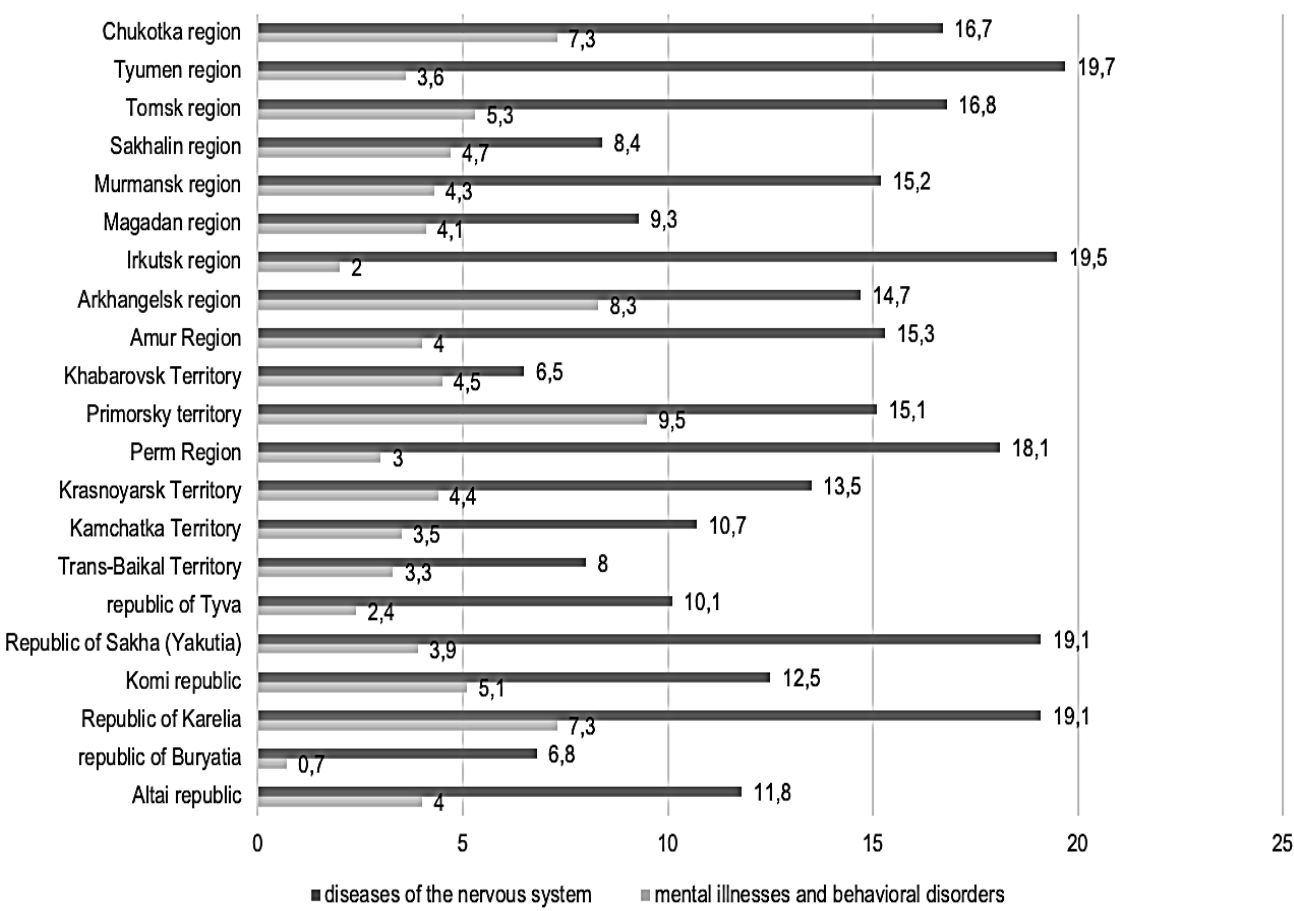

Figure 7 The number of mental illnesses and diseases of the nervous system by territory, 2019

and the Far East are clearly insufficient in the scientific literature. They are often fragmentary and represent the situation in individual regions - the Republic of Sakha (Yakutia), Krasnoyarsk Territory, Khanty-Mansi and Yamalo-Nenets Autonomous Areas. There are very few works that investigate the health and quality of life of the indigenous minorities of the North, Siberia and the Far East, specialized training of medical personnel for these regions. 


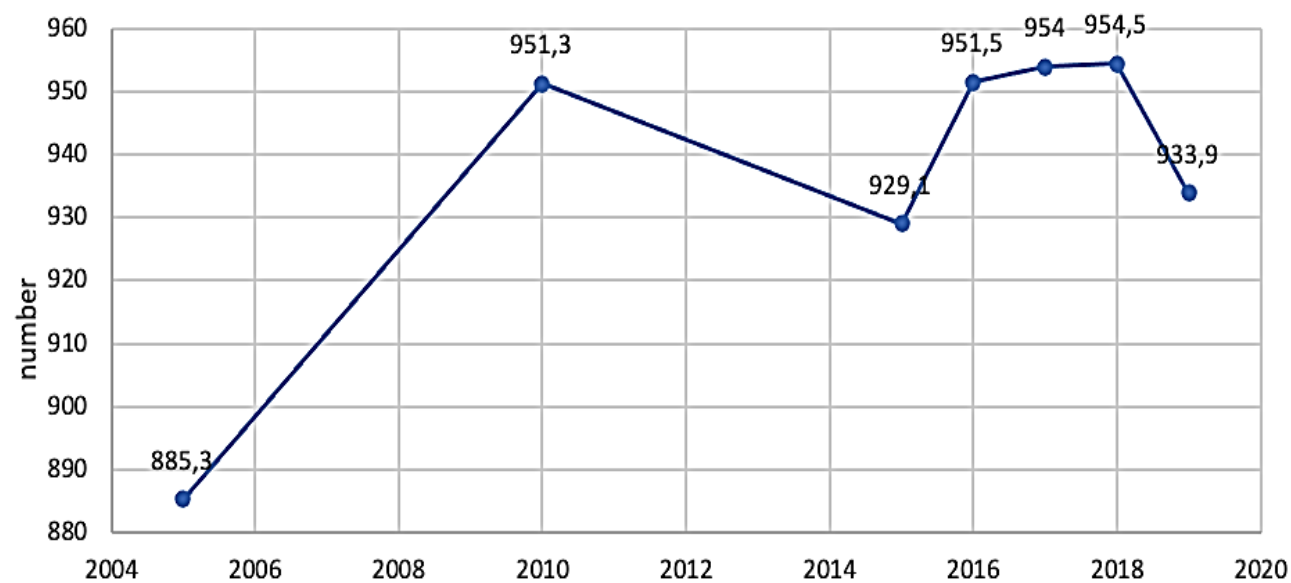

Figure 8 Number of registered diseases, 2004-2019

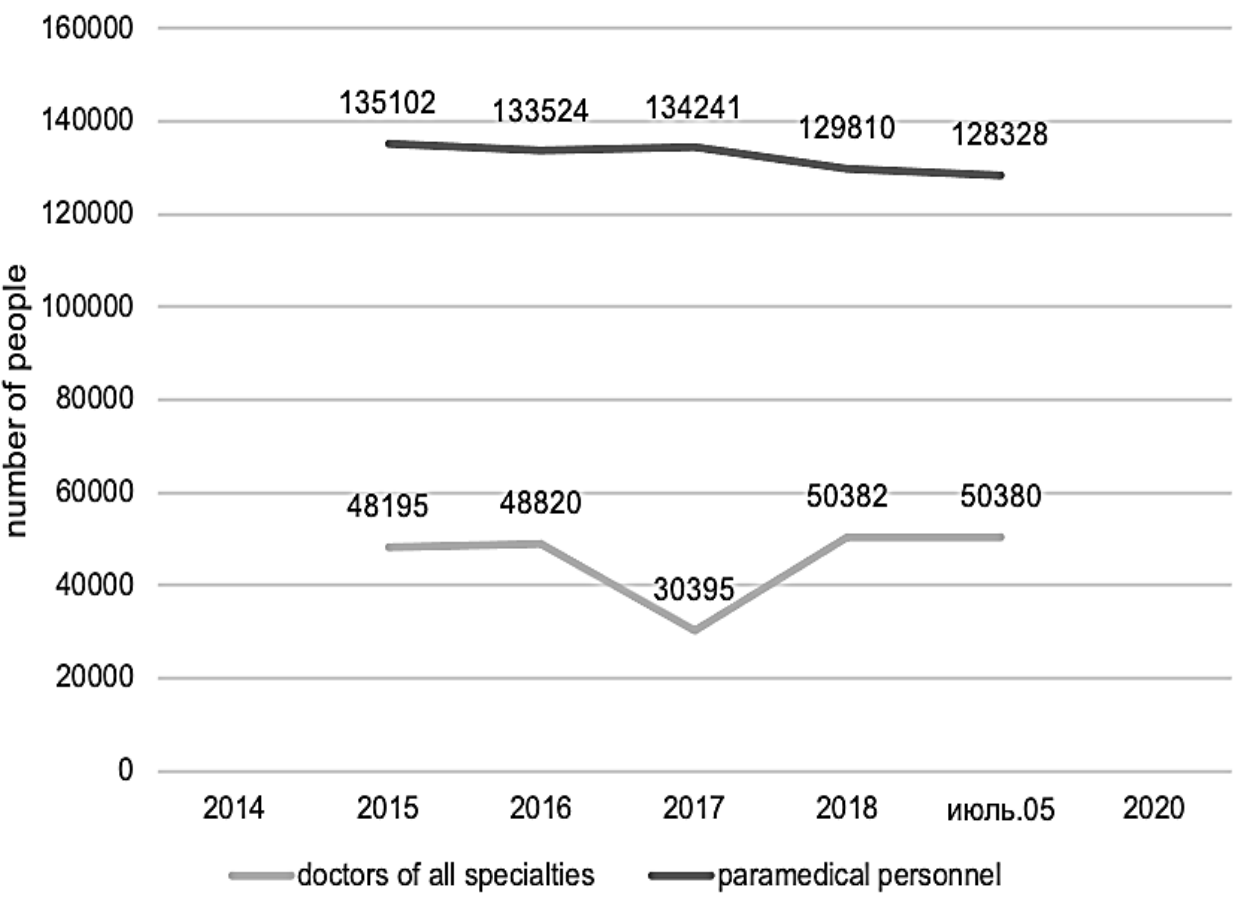

Figure 9 Number of medical personnel, 2014-2019

Statistical accounting can adequately reflect the situation in regions where the indigenous minorities of the North, Siberia and the Far East make up most of the population, but in other cases, the information obtained should be considered as indirect, additional research is required such as obtaining missing data, clarification and verification already available information.

In addition, the complexity of such accounting and analysis includes the fact that many representatives of the indigenous minorities of the North, Siberia and the Far East are nomadic, moving from one administrative region to another without any registration.

\section{RESULTS AND DISCUSSION}

The problem of collecting analytical information using statistical data distributed according to the territorial principle is that the officially established boundaries of the territories do not always include (and, accordingly, take into account) the settlements - the places of permanent residence of the indigenous minorities of the North, Siberia and the Far East. Accordingly, these territories, regardless of the residence of the representatives of the indigenous minorities, are not subject of targeted support programs for this category of the population. 


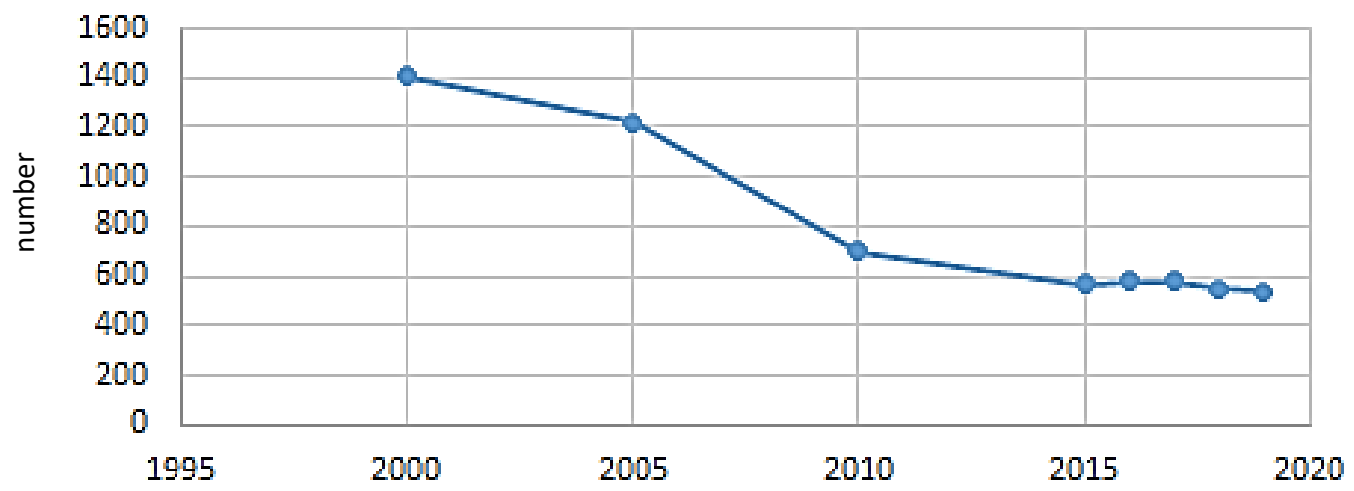

Figure 10 Number of hospitals, 2000-2019

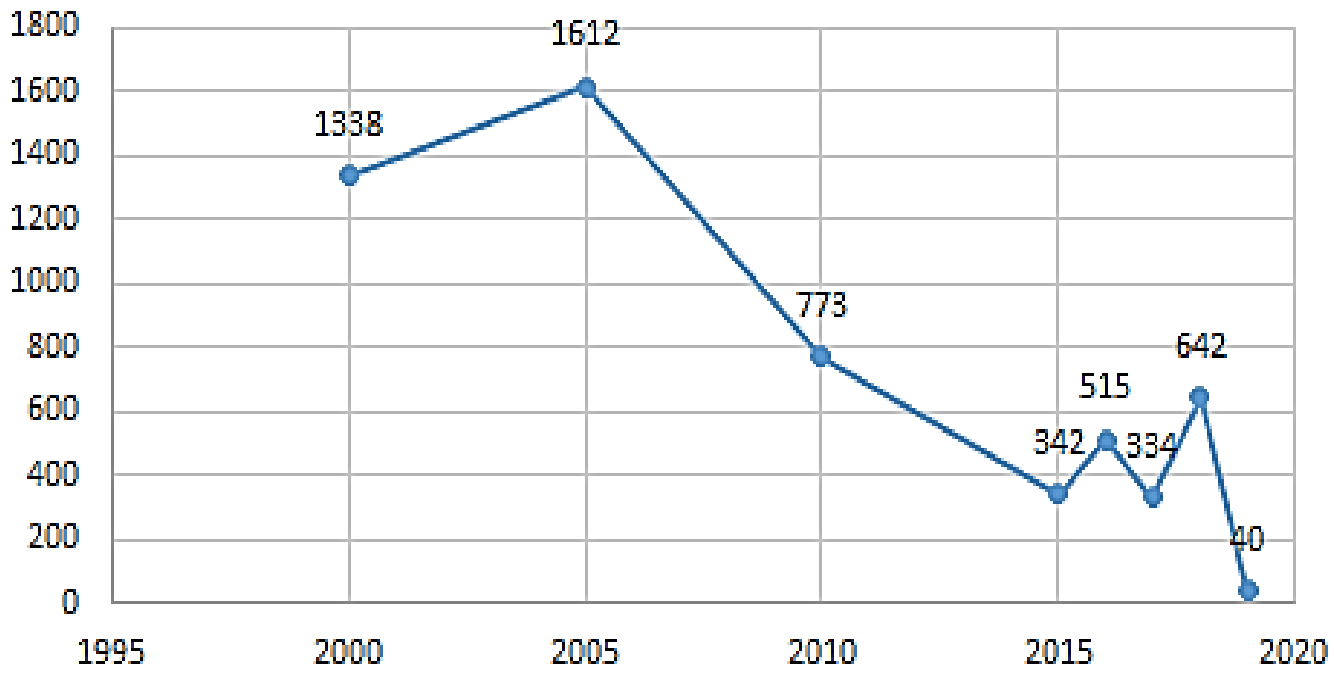

Figure 11 Hospital facilities capacity, 2019

Thus, the existing system of state statistics does not provide the necessary coverage, completeness and reliability of data about the indigenous minorities of the North, Siberia and the Far East; it is impossible to build full-fledged analytical reviews about their quality of life and develop appropriate long-term programs solely on its basis. When making decisions based only on this data, the governing bodies are forced to use partially inaccurate and substantially incomplete information.

Comparing research information with statistical data, one can find the main trends associated with the demographic situation in the northern regions of the Russian Federation (Fig. 1).

Changes in the demographic situation are traditionally determined by the magnitude of natural and mechanical growth. The data presented in Fig. 2 show the negative derivative of the value of natural increase. This trend began to take shape in 2015, in which the peak value of this indicator was recorded [9].

At the same time, it should be noted that significant progress has been achieved in this region in the field of health care, for example, terms of infant mortality (Fig. 3):

One of the serious social problems of the indigenous minorities of the North, Siberia and the Far East of the Russian Federation is an extremely high level of mortality due to alcoholism, infectious and oncological diseases, and suicides (Fig.4-8) [7].

In general, noting the high incidence rate per 1000 people, it should be noted that compared to 2015 , there was an increase in this indicator, although in 2019 it was slightly lower than in 2018.

The least favorable according to this indicator are the Republic of Karelia, the Chukotka, the Republic of Komi and the Republic of Sakha (Yakutia). A more favorable situation in the Republic of Buryatia and the TransBaikal Territory.

Experts highlight the climatic and regional features of the indigenous minorities of the North, Siberia and the Far East that affect their health and well-being. So, life in conditions where frosts reach extreme levels, leaves an imprint on the health of the indigenous minorities of the 
North, Siberia and the Far East. Experts note the hardness and physical endurance of young people who were born and raised in those places. Experts also note a favorable ecological environment in places of residence, which has a positive effect on the health of young people.

The diet of the indigenous minorities of the North, Siberia and the Far East is also important. The main part of their menu is fish and meat; in summer, berries, mushrooms, wild plants appear in the diet. Changes in diet due to relocation or because of limited access to traditional hunting and fishing places, decline in reindeer husbandry can lead to health problems.

According to experts, the main factor negatively affecting the health of the indigenous minorities of the North, Siberia and the Far East is the low level of access to medicine in places of compact residence, which is a common factor for all residents of this territory.

Obviously, in order to understand the reasons for this situation, these data must be correlated with the indicators of the provision of medical personnel (Fig. 9).

As the statistics and research results of the Research Institute of Arctic Medicine of the Northern State Medical University (Arkhangelsk) show, there are not enough doctors and nurses in the areas where indigenous peoples live, the number of hospital and outpatient clinics, medical and obstetric centers, consultations (Fig. 10) [2]. The situation with the commissioning of the capacities of hospital organizations is extremely unfavorable - the number of commissioned beds is rapidly decreasing (Fig. 11).

This circumstance is particularly alarming, given that young people mainly turn to medical specialists with their health problems: $60,6 \%$ of the indigenous minorities of the North, Siberia and the Far East youth have such experience, which is significantly higher than the youth of other ethnic groups.

More than a third of young people of the indigenous minorities of the North, Siberia and the Far East (42,3\%) have experience of referring to medical specialists in a commercial clinic, and $15,5 \%$ - in the last 12 months.

Attitudes towards seeking help from such specialists as shamans $(7,5 \%)$, herbalists and healers $(6,4 \%)$, people with "extrasensory" abilities (fortune teller, astrologer, etc.) $(3,1 \%)$, homeopaths $(1,6 \%)$, youth of the indigenous minorities of the North, Siberia and the Far East are very negative [5].

\section{CONCLUSION}

Thus, despite the considerable experience and undoubted achievements of government statistics, the use of its data to analyze and assess the quality of life of the indigenous minorities of the North, Siberia and the Far East has significant limitations. Much of the information received allows only indirect conclusions that cannot be used by the executive authorities in making informed decisions and formulating development programs for the indigenous minorities of the North, Siberia and the Far East. The development and assessment of the implementation effectiveness of such programs should be based on specially organized surveys and purposefully collected data directly about the indigenous minorities of the North, Siberia and the Far East, supplementing and refining the data of official state and/or departmental statistics. In addition, the methodology of data collection itself needs to be revised, since in the current situation the information collected reflects the situation for only about $70 \%$ of the representatives of the the indigenous minorities of the North, Siberia and the Far East of their actual number.

Note that this is not a characteristic of exclusively Russian research programs; a similar situation has developed in foreign research practice, which pay attention to a lower level of health, without relying on any quantitative data and/or statistical data. It is obvious that it is hardly possible to solve the problem of improving the quality of life of indigenous peoples without serious reliance on monitoring and statistical data.

The topic of health in the modern world is becoming increasingly relevant far beyond the medical context. An important role is played here by the changes taking place in society as a whole. The health of the younger generation and youth is becoming the main priority of a developed society.

Over the past decades, the concept of health has undergone evolution: from considering from the standpoint of the absence of diseases, then to a functional definition of health as a person's ability to fully fulfill their social roles, to an expanded understanding of health as a factor of subjective well-being and the most important indicator of quality of life. It is general wellbeing, rather than the narrowly understood physical health, that becomes the most significant and at the same time the most difficult construct to measure [10].

The strong vulnerability of indigenous peoples, their susceptibility to assimilation, and difficult living conditions put them on the brink of extinction. Many ethnic groups already number only a few hundred people.

Another basis that determines the direction and content of analytical studies on the quality of life of the indigenous minorities of the North, Siberia and the Far East is the understanding that the problems existing in the health care system of them can be divided into general and specific, characteristic only for a certain ethnic group.

Identification of general trends, the specifics of their manifestation in a certain region and a multi-ethnic environment, understanding the specific characteristics 
of health will allow a differentiated approach to data analysis, make more informed conclusions, and make optimal decisions.

These specific features of the indigenous minorities of the North, Siberia and the Far East health care system include limited opportunities for communication, remoteness from medical centers, underdeveloped transport infrastructure, inaccessibility of information sources, low level of knowledge about health preservation, significant differences in living conditions and life, peculiarities of psychophysiological development, shortage of highly qualified medical specialists, etc.

An important aspect that is not practically mentioned in the literature is the health economics of the indigenous minorities of the North, Siberia and the Far East. Obviously, the analysis of both the health costs and the economic effects of it cannot be approached by traditional methods. Thus, it should care about the development of a special methodology and methods of economic analysis of the health care system of the indigenous minorities of the North, Siberia and the Far East at the macro-, meso- and micro-levels.

Monitoring of the quality of life of the indigenous minorities of the North, Siberia and the Far East of the Russian Federation, carried out based on the analysis of statistical data, information sources and websites of the Administrations of regions and municipal districts, revealed common problems associated with the preservation and strengthening of the health of these peoples, regardless of the region of their residence. In some regions, these problems are more significant, in some, less, but everywhere they exist.

\section{ACKNOWLEDGMENTS}

This work was financially supported by the Ministry of Education of the Russian Federation within the framework of a state assignment on the topic "Researching the content and methods of teaching native languages, the content and methods of conducting Olympiads and other competitive events in the native languages of the indigenous peoples of the North, Siberia and the Far East of the Russian Federation".

\section{REFERENCES}

[1] Sh. Garipov, The concept of "indigenous people" and their status in international and domestic law, International law and international organizations 3 (2013) pp. 408-420. DOI: https://doi.org/10.7256/2226-6305.2013.3.5362.

[2] L. N. Gorbatova, G. N. Degteva, L. A. Zubov, Arctic medicine: problems and prospects. Arctic Vedomosti 3 (2015) pp. 74-79.
[3] S. N. Gorbunov, M. Yu. Zadorin, Indigenous peoples and sustainable development: monogr. SAFU 2014 p. 357.

[4] J.J.K. Jaakkola, S. Juntunen, K. Näkkäläjärvi, The Holistic Effects of Climate Change on the Culture, Well-Being, and Health of the Saami, the Only Indigenous People in the European Union. Current Environmental Health Reports 2018. DOI: https://doi.org/10.1007/s40572-018-0211-2

[5] N. G. Khairullina, The problem of alcoholism among the indigenous peoples of the North. Modern problems of science and education 1 (2015).

[6] V.N. Leksin, B.N. Porfiriev, The Russian Arctic: The Logic and Paradoxes of Change. Studies on Russian Economic Development volume 2019. DOI: https://doi.org/10.1134/S1075700719060108.

[7] A.V. Melnikov, Medical and demographic issues of the indigenous population of the western territories of the Khanty-Mansi Autonomous Okrug-Yugra. Demographic potential of the EAEU countries: tez. dokl. 8th Ural Demographic Forum, 2017 pp. 429433.

[8] B. Poppel, Well-Being of Circumpolar Arctic Peoples: The Quest for Continuity. The Pursuit of Human Well-Being. Springer, International Handbooks of Quality-of-Lifeб 2017. DOI: https://doi.org/10.1007/978-3-319-39101-4_17.

[9] V. A. Tishkov, N. I. Novikova, E. A. Pivneva, Indigenous peoples of the Russian Arctic. Bulletin of the Russian Academy of Sciences 85(5-6) (2015) pp. 491 - 500. DOI: https://doi.org/10.7868/S08695 87315060328 .

[10] F. Wu, Modern Economic Growth, Culture, and Subjective Well-Being: Evidence from Arctic Alaska. Journal of Happiness Studies 2021. DOI: https://doi.org/10.1007/s10902-020-00335-4. 